\title{
Unicompartmental vs. segmental bicompartmental vs. total knee replacement: comparison of clinical outcomes
}

Oday Al-Dadah ${ }^{1,2^{*}}$ (D, Georgina Hawes ${ }^{3}$, Philip J. Chapman-Sheath ${ }^{3}$, John William Tice ${ }^{3}$ and David S. Barrett ${ }^{3,4}$

\begin{abstract}
Purpose: Combined medial tibiofemoral and symptomatic patellofemoral osteoarthritis is not amenable to unicompartmental knee replacement (UKR). Total knee replacement (TKR) is an invasive option in younger adults with high functional demands. The aim of this study was to compare the clinical outcome of patients who have undergone UKR, bicompartmental knee replacement (BKR) and TKR up to 2 years post-operatively.

Materials and methods: This prospective study comprised 133 subjects including 30 patients in the medial UKR group, 53 patients in the BKR group (combined medial UKR with patellofemoral joint replacement) and 50 patients in the TKR group. All subjects were evaluated using the Oxford Knee Score (OKS) and the Western Ontario and MacMaster Universities Osteoarthritis Index (WOMAC). Patients in each group were assessed using both scoring systems pre-operatively and 6 months, 1 year and 2 years post-operatively.

Results: Significant improvement of OKS was found at 6 months compared to baseline for UKR (22.7 to 38.1, $p=$ 0.046), BKR (22.6 to $36.8, p<0.001$ ) and TKR (16.6 to 34.5, $p<0.001$ ). Significant improvement was also found for the WOMAC sub-scores for all three groups during this time period. After 6 months, there was no further statistically significant improvement in either outcome score in any of the groups up to the 2-year follow-up results. There was no significant difference in either outcome score post-operatively between the three groups.

Conclusion: The magnitude of clinical improvement following knee replacement is greatest at 6 months; thereafter, only modest improvements continue to occur. This study also found no significant differences of outcomes at 2 years after surgery among UKR, BKR and TKR. BKR is a good alternative option for combined symptomatic medial and patellofemoral arthritis of the knee.
\end{abstract}

Keywords: Bicompartmental, Unicompartmental, Total knee replacement, Arthroplasty, Clinical outcome scores

\footnotetext{
* Correspondence: odayaldadah@hotmail.com

${ }^{1}$ Translational and Clinical Research Institute, Newcastle University,

Framlington Place, Newcastle-upon-Tyne NE2 4HH, UK

2Department of Trauma and Orthopaedic Surgery, South Tyneside Hospital, Harton Lane, South Tyneside NE34 OPL, UK

Full list of author information is available at the end of the article
}

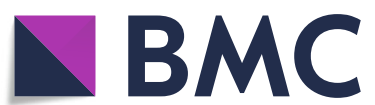

Part of Springer Nature
(9) The Author(s). 2020 Open Access This article is licensed under a Creative Commons Attribution 4.0 International License, which permits use, sharing, adaptation, distribution and reproduction in any medium or format, as long as you give appropriate credit to the original author(s) and the source, provide a link to the Creative Commons licence, and indicate if changes were made. The images or other third party material in this article are included in the article's Creative Commons licence, unless indicated otherwise in a credit line to the material. If material is not included in the article's Creative Commons licence and your intended use is not permitted by statutory regulation or exceeds the permitted use, you will need to obtain permission directly from the copyright holder. To view a copy of this licence, visit http://creativecommons.org/licenses/by/4.0/ The Creative Commons Public Domain Dedication waiver (http://creativecommons.org/publicdomain/zero/1.0/) applies to the data made available in this article, unless otherwise stated in a credit line to the data. 


\section{Introduction}

The management of young adults with symptomatic osteoarthritis of the knee that is refractory to conservative measures is challenging. Total knee replacement (TKR) can successfully treat arthritis albeit at the expense of bone stock and intra-articular ligaments. This can represent theoretical disadvantages, particularly for young patients with high functional demands and high risk for potential revision surgery within their lifetime. Unicompartmental knee replacement (UKR) has been shown to be an effective surgical option in patients with localised disease confined to one compartment that can obviate the need for TKR, with studies reporting up to $91 \%$ survivorship at 20 years [1]. However, medial tibiofemoral compartment arthritis in the presence of generalised patellofemoral joint (PFJ) arthritis (particularly involving the lateral patellar facet) yields poorer clinical results when treated by UKR alone [2, 3]. Furthermore, studies have shown that the main indication for revision of UKR is progression of PFJ arthritis [4, 5]. Arthritis progression following UKR surgery can be minimised by a careful patient selection criteria and avoiding deformity overcorrection in the coronal plane [6]. PFJ replacement also has a role in the management of isolated PFJ arthritis. Although earlier PFJ prostheses were noted to have poor outcomes [7] more recent studies have shown substantially better results [8-10]. One of the commonest indications for PFJ revision surgery is osteoarthritic progression of the tibiofemoral compartments [7, 11]. The improvement of PFJ replacement surgery is attributable to improved implant designs, better patient selection and improved surgical technique [12].

Bicompartmental osteoarthritis disease pattern is a relatively common finding in the knee [13]. When the disease process involves more than one compartment then the situation becomes less favourable for UKR or PFJ replacement alone. These patients may be better served by a segmental bicompartmental knee replacement (BKR). Bicompartmental arthroplasty (a combination of UKR plus PFJ replacement) is less invasive than TKR and is a bone-stock and cruciate-ligamentsparing procedure. This may give a more 'physiological' knee that more closely approximates normal kinematics [13]. BKR can be a successful approach to prevent or postpone the need for TKR. However, this intervention is technically demanding and requires training and experience in both UKR and PFJ replacement surgery. Revision of TKR is a particularly invasive procedure that requires the use of large revision implants. Revision of BKR is comparatively easier as conversion to a standard primary TKR is an available fallback option if needed [14]. This might make BKR an attractive alternative, especially for the young active patient with a high likelihood of future revision.
The literature to date is sparse regarding clinical outcome data of BKR. The limited available literature has been complicated by studies coalescing the results of combined medial and lateral UKRs, medial UKR plus PFJ replacement, lateral UKR plus PFJ replacement and monolithic (femoral monobloc) prostheses all being analysed together. Previous reports $[11,13,15,16]$ of this concept of partial replacement surgery have suggested a significant difference in the survival between these different surgical procedures. We propose a separate modular bicompartmental classification of biunicompartmental (combined medial and lateral UKRs), medial segmental (medial UKR plus PFJ replacement) and lateral segmental (lateral UKR plus PFJ replacement) to accurately distinguish between these different surgical techniques and allow greater clarification in the literature when comparing results of studies.

The aim of this study was to compare the clinical outcome of patients who have undergone medial UKR, medial segmental BKR and TKR. We hypothesised that the outcome of BKR would be similar to that of UKR and TKR.

\section{Material and methods \\ Patients}

The study was a prospective, comparative cohort study assessing the clinical outcomes of patients who underwent UKR, BKR and TKR in our department. This study was exempt from Institutional Review Board (IRB) approval as it was a pragmatic study evaluating the existing clinical practice of each of the surgeons included in the study. There was a total of 133 subjects included in the study. This comprised 30 patients in the UKR group, 53 patients in the BKR group and 50 patients in the TKR group. Table 1 shows the demographics for the subjects in each group. Written informed consent was obtained from all the participants. All patients who underwent surgery had symptomatic osteoarthritis that was refractory to prior conservative treatment. All cases involved unilateral surgery. All patients were mobilised full weight-bearing with full range of movement (ROM) as tolerated and underwent a standardised post-operative physiotherapy rehabilitation programme. All the cases included in this study had their surgery performed under

Table 1 Demographics of subjects

\begin{tabular}{llll}
\hline & $\begin{array}{l}\mathrm{UKR}^{\mathrm{a}} \\
(n=30)\end{array}$ & $\begin{array}{l}\mathrm{BKR}^{\mathrm{b}} \\
(n=53)\end{array}$ & $\begin{array}{l}\mathrm{TKR}^{\mathrm{c}} \\
(n=50)\end{array}$ \\
\hline Mean age (years) (SD) & $60(11)$ & $55(9)$ & $59(7)$ \\
Male: female & $13: 17$ & $22: 31$ & $16: 34$ \\
Index knee (right: left) & $17: 13$ & $31: 22$ & $26: 24$ \\
\hline
\end{tabular}

${ }^{a} U K R$ unicompartmental knee replacement group

${ }^{b} B K R$ bicompartmental knee replacement group

${ }^{\mathrm{C}} T K R$ total knee replacement group 
the care of one of three fellowship trained consultant orthopaedic surgeons with a specialist interest in knee surgery who worked in a tertiary referral university hospital. There were no revision surgical procedures in any of the three groups within the study time period from 2009 to 2012.

The patients in the UKR group had isolated grade-IV medial tibiofemoral arthritis with intact cruciate ligaments and no associated articular cartilage lesions within the remaining two compartments of the knee greater than grade II (superficial fibrillation) of the modified Outerbridge classification [17-19]. The medial UKR prostheses (selected by individual surgeon's preference) included the Sigma High Performance (HP) UKR (DePuy, Warsaw, IN, USA) and the High Flex UKR (Zimmer, Warsaw, IN, USA). Both prostheses are cemented with a metal-backed, fixed-bearing tibial component. All UKR procedures were performed through a minimally invasive, quadriceps-sparing surgical approach.

Similarly, the BKR group had intact cruciate ligaments but with grade-IV arthritis affecting both the medial tibiofemoral and patellofemoral compartments but with well-preserved articular cartilage (no greater than grade II) of the lateral tibiofemoral compartment (Fig. 1). They underwent simultaneous segmental medial UKR and PFJ replacement at the same primary surgical procedure. The indication for PFJ replacement included grade-IV arthritis visible on skyline view $\mathrm{x}$-ray with associated anterior knee pain, palpable crepitus and a positive patellar grind test. The patients who received the Sigma HP UKR prosthesis also received the Sigma HP PFJ replacement (DePuy, Warsaw, IN, USA) (Fig. 2). The patients who received the High Flex UKR prosthesis also received the Gender Solutions PFJ replacement (Zimmer, Warsaw, IN, USA) (Fig. 3). Both PFJ prostheses consisted of cemented trochlea and polyethylene patella resurfacing components. All BKR procedures were performed through a medial parapatellar surgical approach using a notably smaller skin incision than that for TKR.

The TKR group had widespread grade-IV degenerative changes affecting all three compartments of the knee. The TKR prostheses (selected by individual surgeon's preference) included the PFC Sigma HP TKR (DePuy, Warsaw, IN, USA) and the NexGen TKR (Zimmer, Warsaw, IN, USA). Both were cemented, fixed-bearing, posterior-stabilised prostheses. All patients in the TKR group also underwent cemented polyethylene patella resurfacing to minimise possible confounding factors as compared to the BKR group in particular. All TKR procedures were performed through a standard medial parapatellar surgical approach.

\section{Patient-reported outcome measures (PROMs)}

Patients in all three groups were assessed using two validated clinical outcome knee scoring systems preoperatively and 6 months, 1 year and 2 years postoperatively. These included the Oxford Knee Score (OKS, using the new (0-48) scoring system) $[20,21]$ and the Western Ontario and MacMaster Universities Osteoarthritis Index (WOMAC) [22].

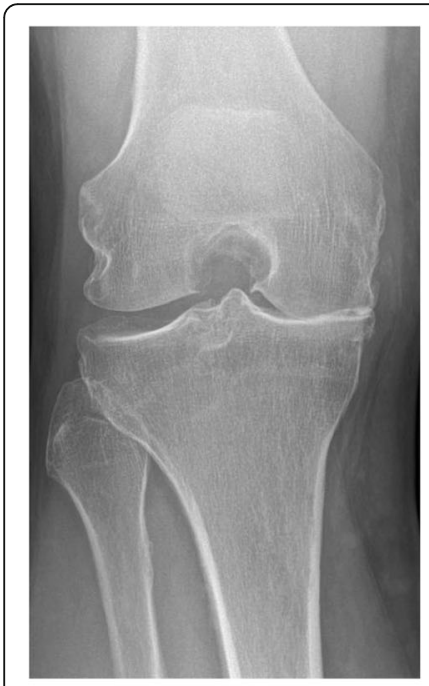

A
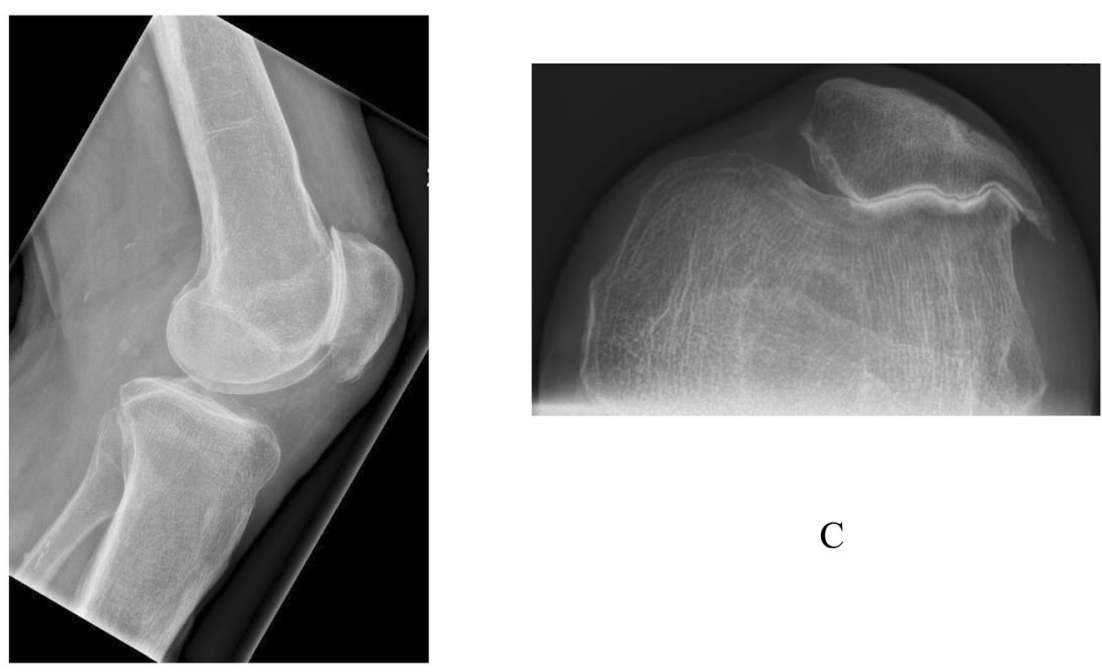

B

Fig. 1 Knee radiographs of ideal candidate for medial segmental bicompartmental knee replacement. a Rosenberg b Lateral c Skyline 


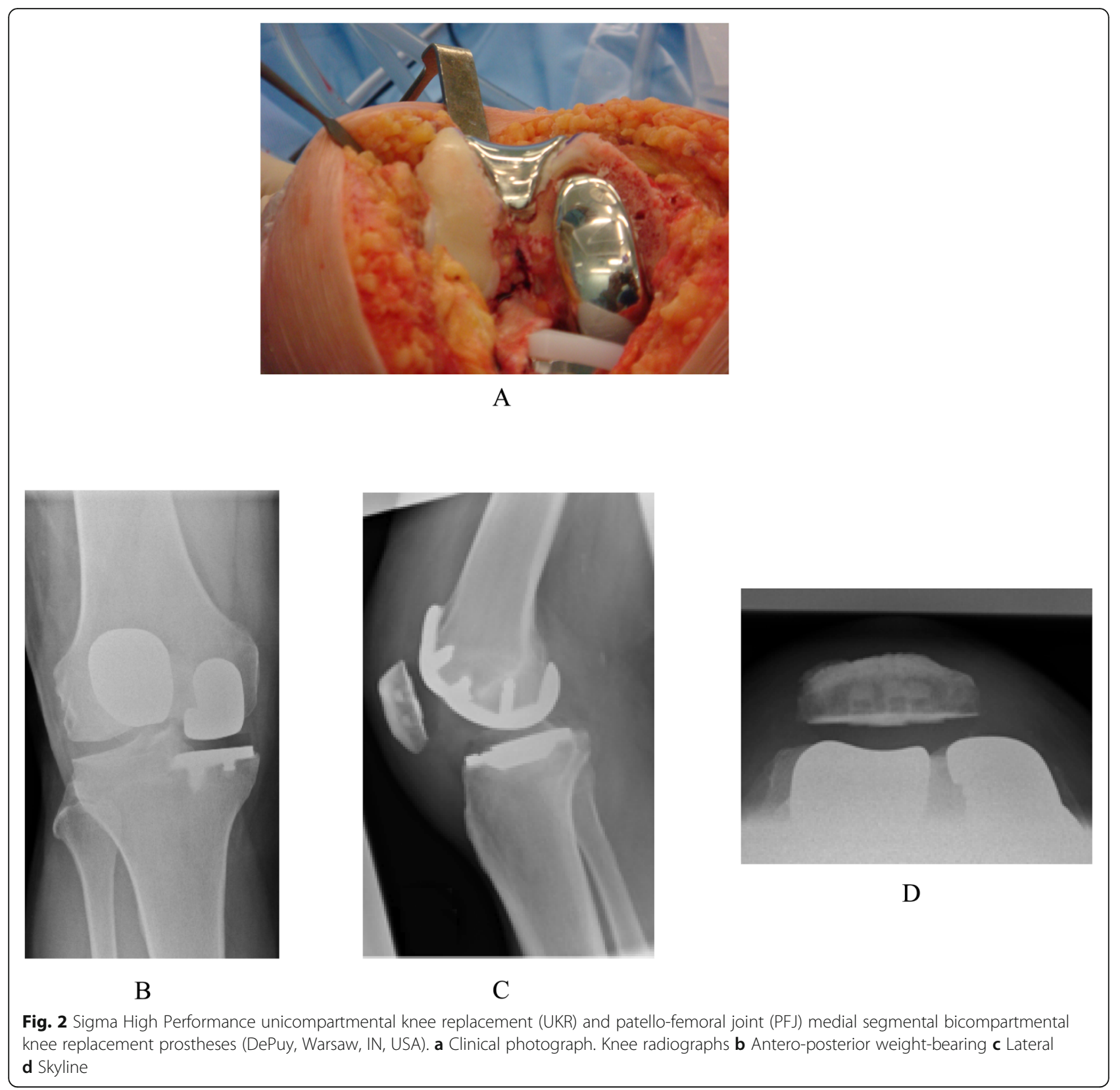

\section{Statistical analysis}

Plotted histograms and the Kolmogorov-Smirnov test were used to confirm that a Normal distribution was an appropriate assumption for all the variables in the study. One-way analysis of variance (ANOVA) and Tukey's post-hoc pairwise comparison was used for the purpose of between-group statistical analyses. The paired Student's $t$ test was used for the longitudinal within-group analyses. The level of statistical significance was set at $p<0.05$. Statistical analysis was performed using SPSS for Windows version 20.0 (IBM Corp., Armonk, New York).

\section{Results}

\section{Oxford Knee Score}

The mean scores of the OKS for each of the three groups are displayed in Fig. 4. The results of the longitudinal within-group statistical analysis in Table 2 revealed a significant improvement of the mean 6month post-operative results as compared to the baseline pre-operative findings for all three groups. After 6 months there was no further statistically significant longitudinal improvement within any of the groups up to the 2-year follow-up results. It was not possible to perform the analysis for the UKR group at the 1-year 


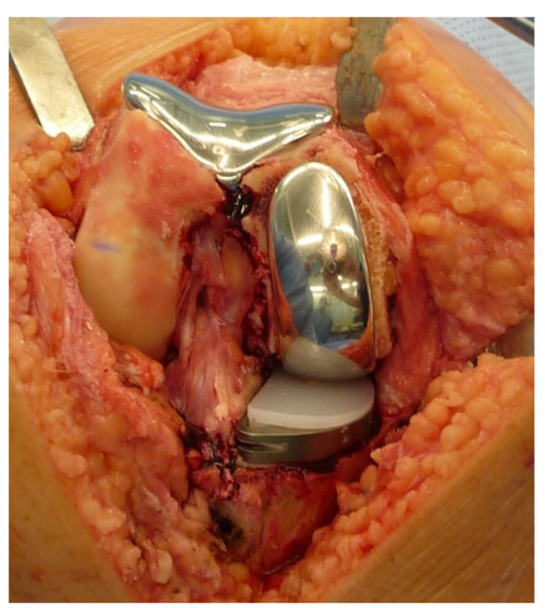

A

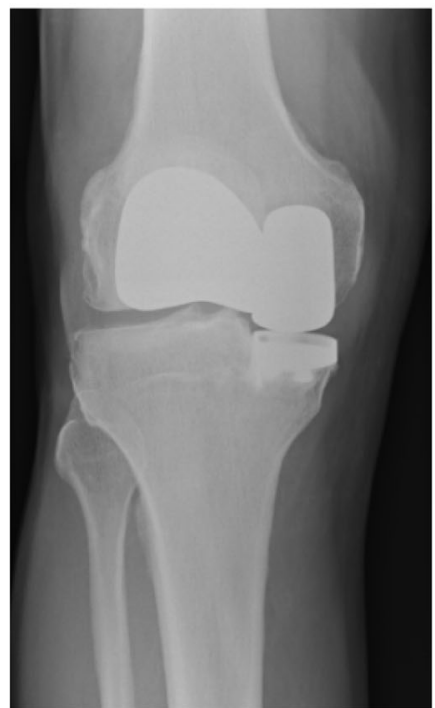

B

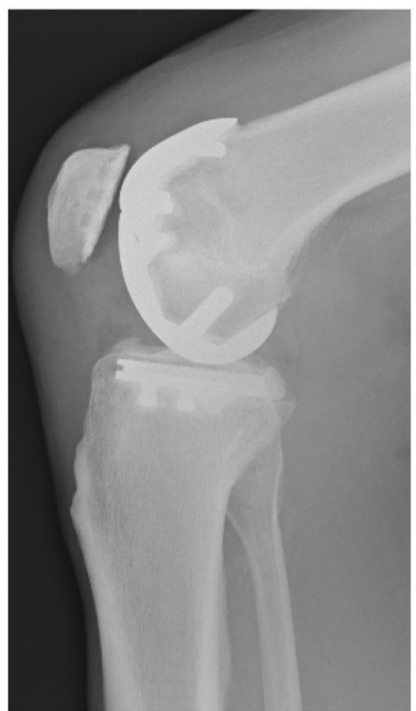

$\mathrm{C}$

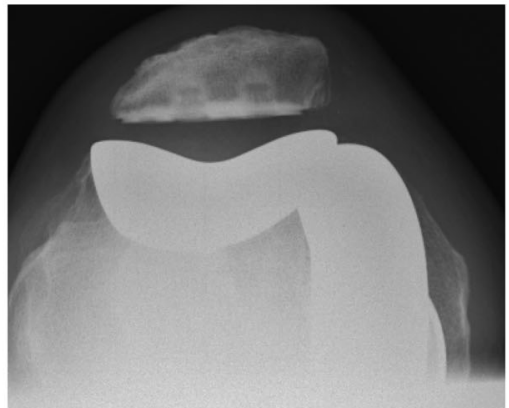

$\mathrm{D}$

Fig. 3 High Flex unicompartmental knee replacement (UKR) and Gender Solutions patello-femoral joint (PFJ) medial segmental bicompartmental knee replacement prostheses (Zimmer, Warsaw, IN, USA). a Clinical photograph. Knee radiographs b Antero-posterior weight-bearing $\mathbf{c}$ Lateral d Skyline

vs. 2-year time point as it was underpowered due to missing data points and loss to follow-up. The between-group statistical analysis shown in Table 3 revealed that pre-operatively there was no significant difference of the OKS between the UKR group and the BKR group. However, there was a significant difference when the TKR group was compared to the UKR group and also the BKR group pre-operatively. Postoperatively there was no significant difference of the mean OKS between any of the three groups at any time point.

\section{Western Ontario and MacMaster Universities Osteoarthritis Index (WOMAC)}

The mean WOMAC sub-scores for each of the three groups is displayed in Fig. 5. The results of the longitudinal within-group statistical analysis in Table 4 revealed a significant improvement of the mean 6-month postoperative results as compared to the baseline preoperative findings for the BKR and TKR groups. The UKR group revealed a borderline significant improvement of the pain sub-score only in this respect. A typeII statistical error may account for the discrepancy of 


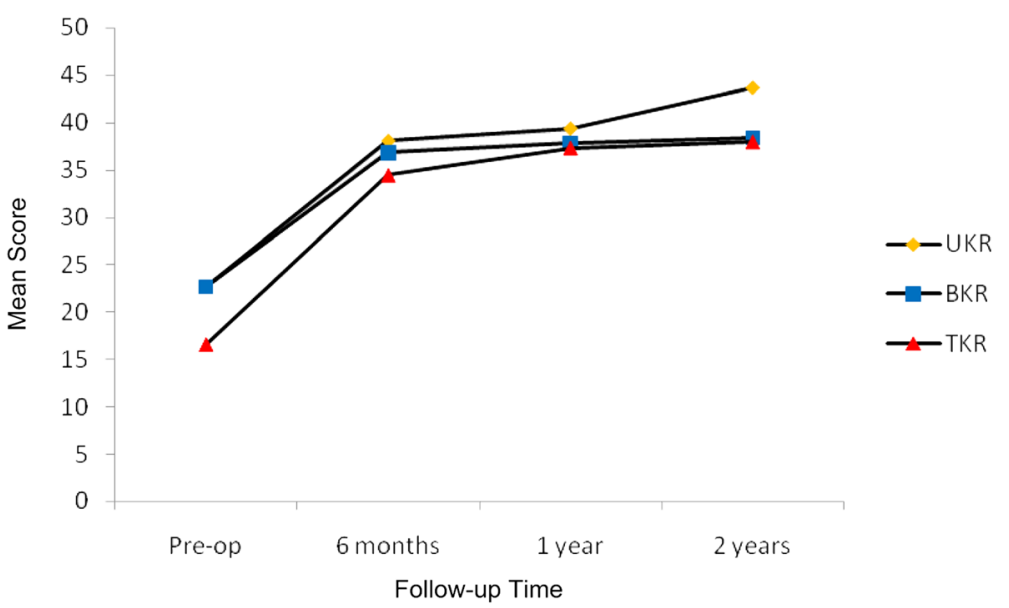

Fig. 4 Mean Oxford Knee Scores. Significant improvement of 6-month post-operative results as compared to pre-operative findings in all three groups. Thereafter, no further statistically significant longitudinal improvement within any of the groups at any time point

the 6-month results for the UKR group. After 6 months there was no further statistically significant longitudinal improvement within any of the groups up to the 2-year follow-up results with the exception of the pain subscore for the UKR group at the 1-year vs. 2-year time point. The between-group statistical analysis shown in Table 5 revealed that pre-operatively there was only a significant difference between the UKR group and the TKR group for the function sub-score. Post-operatively there was no significant difference of the mean WOMAC sub-scores between any of the three groups at any time point.

\section{Discussion}

The principle finding of this study is that the clinical outcome of medial segmental BKR is comparable to that of both medial UKR and TKR. In general, there was a significant improvement of the clinical outcome scores in all three groups at 6 months following surgery as compared to the pre-operative findings. No further significant improvement was found thereafter up to the 2-year results.
We found that the majority of appreciable clinical improvement occurs within the first 6 months following surgery and subsequent to that only modest improvements continue to occur. Post-operatively no significant difference was found between the three groups in terms of outcome scores. However, pre-operatively the mean scores of the TKR group were poorer than those of the UKR and BKR groups. This can be explained by the fact that the subjects who underwent TKR had more generalised (and, therefore, symptomatically more pronounced) arthritic changes in their knee as compared to the more localised and confined disease pattern considered suitable for UKR and BKR intervention.

The use of patient-reported outcome measures (PROMs) is strongly encouraged by the Department of Health [23] as a 'means of assessing effectiveness of care'. Their use is important in both clinical practice and clinical research for measuring improvement in pain and function after surgery and for the comparison of different clinical interventions [24]. We used two validated patient-completed instruments in our study. Clinician-

Table 2 Within-group statistical analysis of Oxford Knee Scores

\begin{tabular}{|c|c|c|c|c|c|c|}
\hline & \multicolumn{2}{|c|}{ Pre-operative vs. 6 months } & \multicolumn{2}{|c|}{6 months vs. 1 year } & \multicolumn{2}{|c|}{1 year vs. 2 years } \\
\hline & $p$ value & $95 \% \mathrm{Cl}$ & $p$ value & $95 \% \mathrm{Cl}$ & $p$ value & $95 \% \mathrm{Cl}$ \\
\hline$\overline{U K R^{a}}$ & $0.046^{*}$ & 0.4 to 30.8 & 0.336 & -5.7 to 10.3 & $\mathrm{~N} / \mathrm{A}$ & \\
\hline$B K R^{b}$ & $<0.001^{*}$ & 11.3 to 19.9 & 0.071 & -0.3 to 4.8 & 0.635 & -17.5 to 13.5 \\
\hline$T_{K R} R^{c}$ & $<0.001^{*}$ & 13.6 to 21.9 & 0.535 & -2.2 to 4.1 & 0.078 & -0.3 to 3.5 \\
\hline
\end{tabular}

Paired Student's $t$ test

$95 \% \mathrm{Cl}$ confidence interval of mean difference

N/A not analysed as underpowered

"Statistically significant at $<0.05$ level

a UKR unicompartmental knee replacement group ( 6 months: $n=21 ; 1$ year: $n=15 ; 2$ years: $n=4$ )

${ }^{\mathrm{b}} B K R$ bicompartmental knee replacement group (6 months: $n=39 ; 1$ year: $n=26 ; 2$ years: $n=15$ )

${ }^{C} T K R$ total knee replacement group ( 6 months: $n=41 ; 1$ year: $n=29 ; 2$ years: $n=11$ ) 
Table 3 Between-group statistical analysis of Oxford Knee Scores

\begin{tabular}{|c|c|c|c|c|c|c|}
\hline & \multicolumn{2}{|c|}{$\mathrm{UKR}^{\mathrm{a}}$ vs. BKR } & \multicolumn{2}{|c|}{ UKR ${ }^{a}$ vs. TKR } & \multicolumn{2}{|c|}{$\mathrm{BKR}^{\mathrm{b}}$ vs. TKR } \\
\hline & $p$ value & $95 \% \mathrm{Cl}$ & $p$ value & $95 \% \mathrm{Cl}$ & $p$ value & $95 \% \mathrm{Cl}$ \\
\hline Pre-operative & 1.000 & - 5.8 to 5.9 & $0.012^{*}$ & 1.1 to 11.1 & $0.013^{*}$ & 1.1 to 11.0 \\
\hline 6 months & 0.917 & -6.7 to 9.3 & 0.461 & -3.7 to 10.9 & 0.641 & -8.4 to 3.8 \\
\hline 1 year & 0.899 & -7.1 to 10.3 & 0.824 & -6.3 to 10.3 & 0.986 & -6.3 to 7.2 \\
\hline 2 years & 0.497 & -6.4 to 17.0 & 0.478 & -6.5 to 17.9 & 0.993 & -8.7 to 8.0 \\
\hline
\end{tabular}

One-way analysis of variance (ANOVA) and Tukey's post-hoc pairwise comparison $95 \% \mathrm{Cl}$ confidence interval of mean difference

"Statistically significant at $<0.05$ level

a UKR unicompartmental knee replacement group (6 months: $n=21 ; 1$ year: $n=15 ; 2$ years: $n=4$ )

${ }^{\mathrm{b}} B K R$ bicompartmental knee replacement group ( 6 months: $n=39 ; 1$ year: $n=26 ; 2$ years: $n=15$ )

${ }^{\mathrm{T}} T K R$ total knee replacement group ( 6 months: $n=41 ; 1$ year: $n=29 ; 2$ years: $n=11$ )

completed outcome scoring systems have the potential for interviewer bias and may also incur cost of resources consumed in terms of clinic and consultation time. The advantages of patient-completed knee scores include a longer time period for patients to answer the questions asked within each item of the instrument and the increased willingness to admit to unfavourable responses as interview bias is obviated. Their main disadvantage is the increased potential for missing data due to omitted responses. The primary weakness of this study was the number of missing data points post-operatively, mainly in the UKR group, due to loss to follow-up which rendered one aspect of the OKS statistical analyses too underpowered to calculate. However, the use of two validated scoring systems and the relatively high number of subjects included overall in the study (particularly in the BKR group) meant that enough data was present to adequately investigate the principle aim of the study. Loss to follow-up is common in clinical studies and can be due to many reasons including patients relocating away from the original hospital in which they had their surgery (due to retirement or moving away for work commitments) while other patients may feel that their affected knee has substantially improved and no longer have time to take off work and attend the hospital for clinic review. Other limitations of the study include different disease pattern involvement pre-operatively which then led to the use of different implants as well as non-randomisation of the subjects. However, this was a pragmatic clinical study in which patients underwent the procedure considered most appropriate for them in terms of disease severity and pattern involvement of their knee and also the prosthesis with which the operating surgeon had the most experience with. The followup time period was up to 2 years post-operatively. The findings of this study should, therefore, be interpreted as the short-term clinical results of a large series of medial segmental BKRs. The outcome variables of interest measured in the present study focussed on clinical outcome scoring systems and revision rates. Future studies may

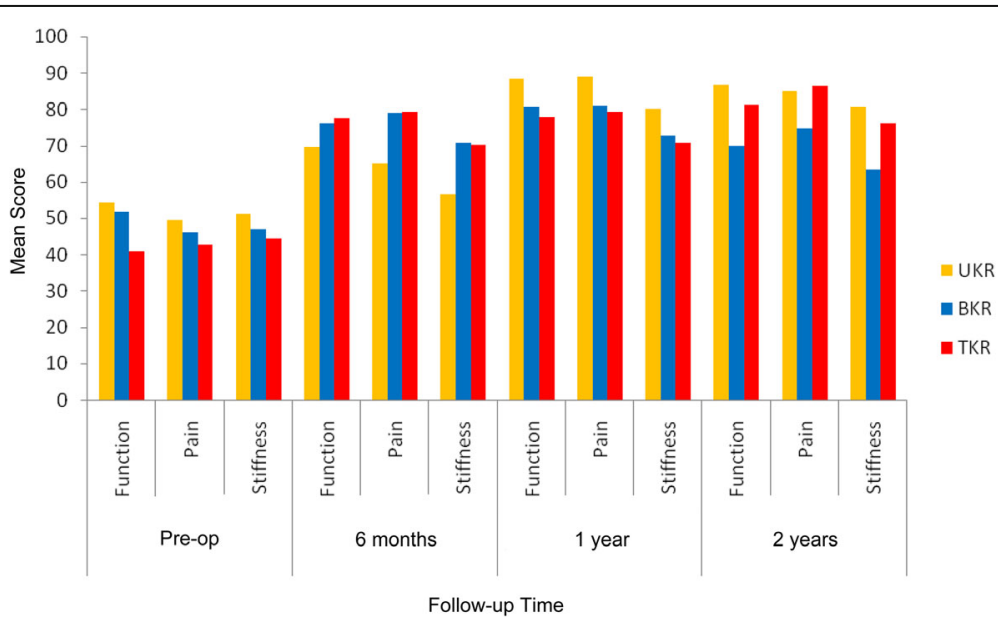

Fig. 5 Mean Western Ontario and MacMaster Universities Osteoarthritis Index (WOMAC) sub-scores. Overall a significant improvement of 6-month post-operative results as compared to pre-operative findings for the bicompartmental knee replacement (BKR) and total knee replacement (TKR) groups but not the unicompartmental knee replacement (UKR) group. Thereafter, no further statistically significant longitudinal improvement generally within any of the groups at any time point 
Table 4 Within-group statistical analysis of Western Ontario and MacMaster Universities Osteoarthritis Index (WOMAC) sub-scores

\begin{tabular}{|c|c|c|c|c|c|c|}
\hline & \multicolumn{2}{|c|}{ Pre-op vs. 6 months } & \multicolumn{2}{|c|}{6 months vs. 1 year } & \multicolumn{2}{|c|}{1 year vs. 2 years } \\
\hline & $p$ value & $95 \% \mathrm{Cl}$ & $p$ value & $95 \% \mathrm{Cl}$ & $p$ value & $95 \% \mathrm{Cl}$ \\
\hline \multicolumn{7}{|l|}{$\mathrm{UKR}^{\mathrm{a}}$} \\
\hline Function & 0.178 & - 96.9 to 169.9 & 0.520 & -194.2 to 225.2 & 0.410 & -2.8 to 5.8 \\
\hline Pain & $0.049^{*}$ & 0.4 to 62.9 & 0.374 & -112.1 to 142.1 & $0.041^{*}$ & 0.3 to 9.7 \\
\hline Stiffness & 0.184 & - 29.0 to 79.3 & 0.500 & -292.7 to 342.7 & 0.750 & -13.3 to -17.3 \\
\hline \multicolumn{7}{|l|}{$B K R^{b}$} \\
\hline Function & $<0.001^{*}$ & 16.4 to 37.1 & 0.249 & -6.8 to 22.3 & 0.172 & -8.0 to 28.5 \\
\hline Pain & $0.001^{*}$ & 17.5 to 45.0 & 0.080 & -0.6 to 8.1 & 0.903 & - 28.8 to 31.3 \\
\hline Stiffness & $0.009^{*}$ & 7.3 to 36.5 & 0.665 & -6.9 to 10.1 & 0.476 & - 19.0 to 32.0 \\
\hline \multicolumn{7}{|l|}{$\mathrm{TKR}^{\mathrm{C}}$} \\
\hline Function & $<0.001^{*}$ & 26.2 to 47.0 & 0.392 & -9.2 to 21.6 & 0.700 & -8.0 to 11.0 \\
\hline Pain & $<0.001^{*}$ & 27.9 to 49.3 & 0.407 & -10.0 to 22.8 & 0.580 & -8.4 to 13.4 \\
\hline Stiffness & $<0.001^{*}$ & 16.4 to 43.3 & 0.385 & -11.4 to 27.0 & 0.306 & -7.7 to 20.1 \\
\hline
\end{tabular}

Paired Student's $t$ test

$95 \% \mathrm{Cl}$ confidence interval of mean difference

"Statistically significant at $<0.05$ level.

a UKR unicompartmental knee replacement group (6 months: $n=25 ; 1$ year: $n=18 ; 2$ years: $n=9$ )

${ }^{\mathrm{b}}$ BKR bicompartmental knee replacement group ( 6 months: $n=41 ; 1$ year: $n=29 ; 2$ years: $n=16$ )

'TKR total knee replacement group ( 6 months: $n=45 ; 1$ year: $n=26 ; 2$ years: $n=14$ )

Table 5 Between-group statistical analysis of Western Ontario and MacMaster Universities Osteoarthritis Index (WOMAC) sub-scores

\begin{tabular}{|c|c|c|c|c|c|c|}
\hline & \multicolumn{2}{|c|}{$U_{K R^{a}}$ vs. BKR } & \multicolumn{2}{|c|}{ UKR ${ }^{a}$ Vs. TKR } & \multicolumn{2}{|c|}{$\mathrm{BKR}^{\mathrm{b}}$ vs. TKR } \\
\hline & $p$ value & $95 \% \mathrm{Cl}$ & $p$ value & $95 \% \mathrm{Cl}$ & $p$ value & $95 \% \mathrm{Cl}$ \\
\hline \multicolumn{7}{|l|}{ Pre-operative } \\
\hline Function & 0.914 & - 11.8 to 16.6 & $0.031^{*}$ & 1.0 to 25.7 & 0.073 & -0.8 to 22.7 \\
\hline Pain & 0.863 & - 13.0 to 18.5 & 0.435 & -6.3 to 19.8 & 0.793 & -9.3 to 16.2 \\
\hline Stiffness & 0.851 & - 14.6 to 23.1 & 0.575 & -9.4 to 22.9 & 0.921 & -13.2 to 18.3 \\
\hline \multicolumn{7}{|l|}{6 months } \\
\hline Function & 0.808 & - 18.3 to 31.1 & 0.706 & - 15.9 to 31.6 & 0.966 & -15.6 to 12.6 \\
\hline Pain & 0.275 & -7.7 to 35.7 & 0.227 & -6.4 to 35.2 & 0.997 & - 13.0 to 13.7 \\
\hline Stiffness & 0.275 & - 7.9 to 36.5 & 0.279 & -7.6 to 34.8 & 0.991 & -14.3 to 12.9 \\
\hline \multicolumn{7}{|l|}{1 year } \\
\hline Function & 0.589 & - 11.2 to 26.5 & 0.349 & -7.6 to 28.4 & 0.920 & -14.2 to 19.7 \\
\hline Pain & 0.498 & - 9.0 to 24.8 & 0.328 & -6.5 to 25.7 & 0.961 & -13.5 to 16.9 \\
\hline Stiffness & 0.686 & - 13.8 to 28.3 & 0.508 & - 10.8 to 29.4 & 0.963 & - 16.9 to 21.0 \\
\hline \multicolumn{7}{|l|}{2 years } \\
\hline Function & 0.197 & -6.7 to 40.6 & 0.850 & - 19.4 to 30.4 & 0.424 & - 10.8 to 33.8 \\
\hline Pain & 0.522 & - 12.8 to 33.6 & 0.990 & - 23.0 to 25.8 & 0.396 & - 10.2 to 33.6 \\
\hline Stiffness & 0.304 & - 11.0 to 45.6 & 0.929 & -25.3 to 34.2 & 0.472 & - 13.9 to 39.5 \\
\hline
\end{tabular}

One-way analysis of variance (ANOVA) and Tukey's post-hoc pairwise comparison

$95 \% \mathrm{Cl}$ confidence interval of mean difference

*Statistically significant at $<0.05$ level.

a UKR unicompartmental knee replacement group (6 months: $n=25 ; 1$ year: $n=18 ; 2$ years: $n=9$ )

${ }^{\mathrm{b}} B K R$ bicompartmental knee replacement group ( 6 months: $n=41 ; 1$ year: $n=29 ; 2$ years: $n=16$ )

'TKR total knee replacement group ( 6 months: $n=45$; 1 year: $n=26 ; 2$ years: $n=14$ ) 
wish to also consider including radiological evaluations, operative time, length of hospital stay and any-cause complications.

UKR is successful in managing patients with single compartment arthritis with intact cruciate ligaments [25]. Both younger and more active patients as well the increasingly ageing population who present with symptomatic osteoarthritis of the knee have high expectations of not only improvement of pain but also physical function following surgery in order to fulfil an active lifestyle after retirement [26]. Some studies have shown that the rate of return to low-impact sporting activities (i.e. golf, bowling, cycling, swimming, etc.) was as high as $96 \%$ following UKR and as low as 63\% following TKR [27]. Patients who underwent UKR were also found to return to sporting activities more quickly (mean 3.6 months) than those who had undergone TKR (mean 4.1 months). UKR and BKR retain both cruciate ligaments (and, therefore, the mechanoreceptors within them) and so preserve and maintain knee proprioception. Patient satisfaction and functional outcome have been closely correlated with knee proprioception [28]. High patient expectations and functional demands following arthroplasty may be met more readily with UKR and BKR than TKR. Approximately half of all patients who have degenerative arthritis of the knee will also have involvement of the PFJ. Symptoms include crepitus and anterior knee pain exacerbated by exercise, kneeling, squatting and stair climbing (particularly ascending stairs) [29]. UKR may be considered in a select group of patients with coexisting but strictly asymptomatic grade-III PFJ arthritis. Intra-operative trimming of the patella with excision of osteophytes or partial lateral facetectomy of the patella can be performed at the time of the UKR operation. However, UKR alone is not advisable in single tibiofemoral compartment arthritis with associated symptomatic and generalised PFJ arthritis [2,3]. Although BKR is a more intricate undertaking than UKR, it is a less invasive surgical option than TKR in patients with associated symptomatic PFJ arthritis [30]. Furthermore, patients who have previously undergone isolated UKR or PFJ replacement and subsequently develop progressive arthritis in an unresurfaced compartment can be effectively managed by staged modular resurfacing of the newly affected compartment (provided the original prosthesis remains functional and well fixed), and thereby forestalling conversion to TKR and preserving a future arthroplasty option [31, 32].

Retaining both cruciate ligaments not only confers a proprioceptive advantage but also preserves the physiological and kinematic function of the knee joint $[33,34]$. Cadaveric studies $[33,34]$ have shown that cruciate-sparing prostheses (i.e. UKR) demonstrate normal kinematics and compressive forces within the
PFJ as compared to the native knee. However, in cruciate-sacrificing prostheses (i.e. TKR) there is loss of the physiological roll-back mechanism of the femur which induces altered kinematics and abnormally high PFJ compressive forces, especially in flexion. This adverse effect was more pronounced in posteriorcruciate-retaining TKR prostheses than posteriorstabilised ones as in the latter the polyethylene tibial peg acted as a mechanical interlock which prevented posterior subluxation of the tibia as the knee flexed. The extensor mechanism is, therefore, vulnerable to the 'knock-on effect' of abnormal tibiofemoral movement, with resultant changes in PFJ forces and tracking. Further in-vitro studies [35] investigating the kinematics of the tibiofemoral joint have shown that the translational and rotational knee-joint kinematics of BKR resemble that of the native knee and were indeed found to be superior to that of posteriorcruciate-retaining TKR. In-vivo studies [36, 37] evaluating knee-joint kinematics throughout a spectrum of activities of daily living motor tasks (i.e. walking, stair climbing, etc.) have shown that knee-joint kinematics in patients with unilateral BKR replicate the kinematics of their contra-lateral non-involved limb and also that of healthy external controls. Wang et al. [38] evaluated knee strength and biomechanics during walking in patients with BKR, TKR and healthy control subjects. They found that BKR patients reported higher satisfaction while performing activities of daily living and improved their quadriceps strength to a level that was close to healthy controls. However, the TKR patients had a reduced walking speed consequent to deficits in quadriceps strength and decreased extensor moment.

There have been variable reports published in the literature regarding the Journey Deuce BKR prosthesis (Smith \& Nephew, Memphis, TN, USA). Its monolithic design (monobloc) consists of a combined femoral shield with a medial condylar component that resurfaces both the medial and patellofemoral (trochlear) compartments of the distal femur. This component is available as either oxidised zirconium or cobalt chrome. The medial tibial component consists of a fixed-bearing, unicondylar, metal-backed prosthesis. The patella can be left either unresurfaced or (more commonly) resurfaced with a polyethylene button [39, 40]. Some studies [40] using this prosthesis demonstrated early discharge from hospital (2 days post-operatively), good range of knee movement, easier post-operative rehabilitation, reduced intra-operative blood loss with no patients requiring blood transfusion, reduced pain and a high level of patient satisfaction. Rolston et al. [41] also showed that the Journey Deuce BKR was effective in terms of restoring the mechanical axis and correct knee alignment to the 
centre of the tibial plateau in $95 \%$ of cases. However, Palumbo et al. [16] reported their experience with the Journey Deuce BKR and found an overall survival rate as low as $86 \%$ at 2 years following surgery and with $31 \%$ of patients unsatisfied due to inconsistent pain relief and unacceptable functional results. Other studies [42] also found significantly higher early complications (mainly persistent pain) that required revision arthroplasty. Muller et al. [43] found that in their series of 43 cases, $18 \%$ of patients had to undergo revision within the first year due to persistent knee pain and instability. The high revision rates in these studies maybe a reflection of the technical complexity of the BKR concept. However, specifically to the Journey Deuce prosthesis itself, adverse factors may include poor implant design, insufficient variety of implant sizes and crude instrumentation. The monobloc femoral component may only allow limited positioning options, thereby making it less accommodating to the variability of the patient's native knee (both in the medial and PFJ compartments) and, therefore, increasing the risk of component malalignment. We used a segmental modular approach (UKR with PFJ replacement) in our study that allowed separate positioning of the implants and so better individual tailoring to the patient's specific knee anatomy. Heyse et al. [14] retrospectively reported on a series of nine BKR cases (segmental UKR with PFJ replacement) at a mean follow-up time period of 12 years with a successful outcome and high patient satisfaction and no revision procedures within the study time frame. Parratte et al. [13] reported on 77 BKR cases (segmental UKR with PFJ replacement) and found that the procedure reliably alleviated pain, improved function and restored limb alignment. The prosthesis survivorship at 17 years was 54\% (27 cases were revised), of which 20 cases were for aseptic loosening of the PFJ prosthesis. Their high revision rates were attributed to the use of early generation implants, poor instrumentation and lack of experience in PFJ replacements at the time of original surgery. Future studies are, therefore, required to assess the long-term outcome of segmental BKR with modern implant designs, improved surgical techniques and appropriate patient selection.

\section{Conclusion}

The magnitude of clinical improvement following knee replacement is greatest at 6 months; thereafter, only modest improvements continue to occur. This study found no significant differences of OKS and WOMAC at 2 years after surgery among UKR, BKR and TKR. BKR is a good alternative option for combined symptomatic medial and patellofemoral arthritis of the knee.

\section{Acknowledgements}

Not applicable

\section{Disclosure of interest}

DSB has a consultancy agreement with DePuy. PJCS has a consultancy agreement with Zimmer. All the other authors declare that they have no conflict of interest.

\section{Authors' contributions}

OA: made substantial contributions to the conception and design of the study and acquisition of data. He also performed the analysis and interpretation of data. He has been involved in drafting the manuscript and revising it critically for important intellectual content. He has given final approval of the version to be published. He also agrees to be accountable for all aspects of the work in ensuring that questions related to the accuracy or integrity of any part of the work are appropriately investigated and resolved. GH: made substantial contributions to the conception and design of the study. She also made substantial contributions to the analysis and interpretation of data. She has been involved in drafting the manuscript and revising it critically for important intellectual content. She has given final approval of the version to be published. She also agrees to be accountable for all aspects of the work in ensuring that questions related to the accuracy or integrity of any part of the work are appropriately investigated and resolved. PJCS: made substantial contributions to conception and design of the study. He also made substantial contributions to the interpretation of data. He has been involved in drafting the manuscript and revising it critically for important intellectual content. He has given final approval of the version to be published. He also agrees to be accountable for all aspects of the work in ensuring that questions related to the accuracy or integrity of any part of the work are appropriately investigated and resolved. JWT: made substantial contributions to conception and design of the study. He also made substantial contributions to the interpretation of data. He has been involved in drafting the manuscript and revising it critically for important intellectual content. He has given final approval of the version to be published. He also agrees to be accountable for all aspects of the work in ensuring that questions related to the accuracy or integrity of any part of the work are appropriately investigated and resolved. DSB: made substantial contributions to conception and design of the study. He also made substantial contributions to the interpretation of data. He has been involved in drafting the manuscript and revising it critically for important intellectual content. He has given final approval of the version to be published. He also agrees to be accountable for all aspects of the work in ensuring that questions related to the accuracy or integrity of any part of the work are appropriately investigated and resolved.

\section{Funding}

No funding was received for this study.

\section{Availability of data and materials}

The datasets used and/or analysed during the current study are available from the corresponding author on reasonable request.

\section{Ethics approval and consent to participate}

All procedures performed in this study involving human participants were in accordance with the ethical standards of the Institutional and/or National Research Committee and with the 1964 Helsinki Declaration and its later amendments or comparable ethical standards. Informed consent was obtained from all individual participants included in the study.

\section{Consent for publication}

Not applicable

\section{Competing interests}

DSB has a consultancy agreement with DePuy. PJCS has a consultancy agreement with Zimmer. All the other authors declare no conflict of interest.

\section{Author details}

'Translational and Clinical Research Institute, Newcastle University, Framlington Place, Newcastle-upon-Tyne NE2 4HH, UK. ${ }^{2}$ Department of Trauma and Orthopaedic Surgery, South Tyneside Hospital, Harton Lane, South Tyneside NE34 OPL, UK. ${ }^{3}$ Department of Trauma and Orthopaedic 
Surgery, University Hospital Southampton, Tremona Road, Southampton, Hampshire SO16 6YD, UK. ${ }^{4}$ School of Engineering Sciences, University of Southampton, Highfield, Southampton SO17 1BJ, UK.

Received: 6 May 2020 Accepted: 14 August 2020

Published online: 31 August 2020

\section{References}

1. Price AJ, Svard U (2011) A second decade lifetable survival analysis of the Oxford unicompartmental knee arthroplasty. Clin Orthop Relat Res 469(1): 174-179

2. Beard DJ, Pandit H, Gill HS, Hollinghurst D, Dodd CA, Murray DW (2007) The influence of the presence and severity of pre-existing patellofemoral degenerative changes on the outcome of the Oxford medial unicompartmental knee replacement. J Bone Joint Surg (Br) 89(12):1597-1601

3. Beard DJ, Pandit H, Ostlere S, Jenkins C, Dodd CA, Murray DW (2007) Preoperative clinical and radiological assessment of the patellofemoral joint in unicompartmental knee replacement and its influence on outcome. J Bone Joint Surg (Br) 89(12):1602-1607

4. Berger RA, Meneghini RM, Sheinkop MB et al (2004) The progression of patellofemoral arthrosis after medial unicompartmental replacement: results at 11 to 15 years. Clin Orthop Relat Res 428:92-99

5. Hernigou P, Deschamps G (2002) Patellar impingement following unicompartmental arthroplasty. J Bone Joint Surg Am 84-A(7):1132-1137

6. Weale AE, Murray DW, Baines J, Newman JH (2000) Radiological changes five years after unicompartmental knee replacement. J Bone Joint Surg (Br) 82(7):996-1000

7. van Wagenberg JM, Speigner B, Gosens T, de Waal Malefijt J (2009) Midterm clinical results of the Autocentric II patellofemoral prosthesis. Int Orthop 33(6):1603-1608

8. Butler JE, Shannon R (2009) Patellofemoral arthroplasty with a custom-fit femoral prosthesis. Orthopedics. 32(2):81

9. Leadbetter WB, Kolisek FR, Levitt RL et al (2009) Patellofemoral arthroplasty: a multi-centre study with minimum 2-year follow-up. Int Orthop 33(6):15971601

10. van Jonbergen HP, Poolman RW, van Kampen A (2010) Isolated patellofemoral osteoarthritis. Acta Orthop 81(2):199-205

11. Confalonieri N, Manzotti A, Montironi F, Pullen C (2008) Tissue sparing surgery in knee reconstruction: unicompartmental (UKA), patellofemoral (PFA), UKA + PFA, bi-unicompartmental (Bi-UKA) arthroplasties. J Orthop Traumatol 9(3):171-177

12. Farr J 2nd, Barrett DS (2008) Optimizing patellofemoral arthroplasty. Knee 15(5):339-347

13. Parratte S, Pauly V, Aubaniac JM, Argenson JN (2010) Survival of bicompartmental knee arthroplasty at 5 to 23 years. Clin Orthop Relat Res 468(1):64-72

14. Heyse TJ, Khefacha A, Cartier P (2010) UKA in combination with PFR at average 12-year follow-up. Arch Orthop Trauma Surg 130(10):1227-1230

15. Confalonieri N, Manzotti A, Cerveri P, De Momi E (2009) Biunicompartmental versus total knee arthroplasty: a matched paired study with early clinical results. Arch Orthop Trauma Surg 129(9):1157-1163

16. Palumbo BT, Henderson ER, Edwards PK, Burris RB, Gutierrez S, Raterman SJ (2011) Initial experience of the Journey-Deuce bicompartmental knee prosthesis: a review of 36 cases. J Arthroplast 26(6 Suppl):40-45

17. Cameron ML, Briggs KK, Steadman JR (2003) Reproducibility and reliability of the outerbridge classification for grading chondral lesions of the knee arthroscopically. Am J Sports Med 31(1):83-86

18. Outerbridge RE (1961) The etiology of chondromalacia patellae. J Bone Joint Surg (Br) 43-B:752-757

19. Outerbridge RE, Dunlop JA (1975) The problem of chondromalacia patellae. Clin Orthop Relat Res 110:177-196

20. Dawson J, Fitzpatrick R, Murray D, Carr A (1998) Questionnaire on the perceptions of patients about total knee replacement. J Bone Joint Surg (Br) 80(1):63-69

21. Murray DW, Fitzpatrick R, Rogers K et al (2007) The use of the Oxford Hip and Knee Scores. J Bone Joint Surg (Br) 89(8):1010-1014

22. Bellamy N, Buchanan WW, Goldsmith CH, Campbell J, Stitt LW (1988) Validation study of WOMAC: a health status instrument for measuring clinically important patient relevant outcomes to antirheumatic drug therapy in patients with osteoarthritis of the hip or knee. J Rheumatol 15(12):1833-1840
23. Department of Health. Guidance on the routine collection of patient reported outcome measures (PROMs). 2012; http://www.dh.gov.uk/en/ Publicationsandstatistics/Publications/PublicationsPolicyAndGuidance/DH_ 092647

24. Marx RG, Jones EC, Atwan NC, Closkey RF, Salvati EA, Sculco TP (2005) Measuring improvement following total hip and knee arthroplasty using patient-based measures of outcome. J Bone Joint Surg Am 87(9):1999-2005

25. Goodfellow JW, Kershaw CJ, Benson MK, O'Connor JJ (1988) The Oxford Knee for unicompartmental osteoarthritis. The first 103 cases. J Bone Joint Surg (Br) 70(5):692-701

26. Nilsdotter AK, Toksvig-Larsen S, Roos EM (2009) Knee arthroplasty: are patients' expectations fulfilled? A prospective study of pain and function in 102 patients with 5-year follow-up. Acta Orthop 80(1):55-61

27. Hopper GP, Leach WJ (2008) Participation in sporting activities following knee replacement: total versus unicompartmental. Knee Surg Sports Traumatol Arthrosc 16(10):973-979

28. Barrett DS (1991) Proprioception and function after anterior cruciate reconstruction. J Bone Joint Surg (Br) 73(5):833-837

29. Donell ST, Glasgow MM (2007) Isolated patellofemoral osteoarthritis. Knee 14(3):169-176

30. Tria AJ Jr (2010) Bicompartmental arthroplasty of the knee. Instr Course Lect 59:61-73

31. Lonner JH (2009) Modular bicompartmental knee arthroplasty with robotic arm assistance. Am J Orthop 38(2 Suppl):28-31

32. Rolston L, Moore C (2010) Conversion of lateral unicompartmental arthroplasty to anterior cruciate retaining tricompartmental knee arthroplasty. Knee 17(3):249-251

33. Miller RK, Goodfellow JW, Murray DW, O'Connor JJ (1998) In vitro measurement of patellofemoral force after three types of knee replacement J Bone Joint Surg (Br) 80(5):900-906

34. Price AJ, Oppold PT, Murray DW, Zavatsky AB (2006) Simultaneous in vitro measurement of patellofemoral kinematics and forces following Oxford medial unicompartmental knee replacement. J Bone Joint Surg (Br) 88(12): 1591-1595

35. Wunschel M, Lo J, Dilger T, Wulker N, Muller O (2011) Influence of bi- and tri-compartmental knee arthroplasty on the kinematics of the knee joint. BMC Musculoskelet Disord 12:29

36. Leffler J, Scheys L, Plante-Bordeneuve T et al (2012) Joint kinematics following bi-compartmental knee replacement during daily life motor tasks. Gait Posture. https://doi.org/10.1016/j.gaitpost.2012.04.008

37. Wang H, Frame J, Rolston L (2012) Influence of bicompartmental knee replacement on stand-to-sit movement. Res Q Exerc Sport 83(2):136-142

38. Wang H, Foster J, Franksen N, Estes J, Rolston L (2018) Gait analysis of patients with an off-the-shelf total knee replacement versus customized bicompartmental knee replacement. Int Orthop 42(4):805-810

39. Engh GA (2007) A bi-compartmental solution: what the Deuce? Orthopedics. 30(9):770-771

40. Rolston L, Bresch J, Engh G et al (2007) Bicompartmental knee arthroplasty: a bone-sparing, ligament-sparing, and minimally invasive alternative for active patients. Orthopedics. 30(8 Suppl):70-73

41. Rolston L, Siewert K (2009) Assessment of knee alignment after bicompartmental knee arthroplasty. J Arthroplast 24(7):1111-1114

42. Morrison TA, Nyce JD, Macaulay WB, Geller JA (2011) Early adverse results with bicompartmental knee arthroplasty: a prospective cohort comparison to total knee arthroplasty. J Arthroplast 26(6 Suppl):35-39

43. Muller M, Matziolis G, Falk R, Hommel H (2012) The bicompartmental knee joint prosthesis Journey Deuce : Failure analysis and optimization strategies. Orthopade 41(11):894-904

\section{Publisher's Note}

Springer Nature remains neutral with regard to jurisdictional claims in published maps and institutional affiliations. 